\title{
Comparación de tasa metabólica en reposo medida por calorimetría indirecta versus ecuaciones predictivas, en mujeres adultas jovenes y adultas mayores de peso corporal normal
}

\author{
Comparison of resting metabolic rate measured by \\ indirect calorimetry and predictive equations \\ in young adult and elderly women with \\ normal body weight
}

\begin{abstract}
Introduction: The resting metabolic rate ( $R M R$ ) can be measured by indirect calorimetry (RMR IC) or estimated by predictive equations, which can overestimate or underestimate energy requirements. Objective: To compare RMRs measured by indirect calorimetry and estimated by predictive equations in young adult and elderly women with normal body mass index (BMI). Subjects and methods: Analytical cross-sectional study. RMR IC was measured and estimated by Harris-Benedict (1919), FAO/WHO/UNU (1985), FAO/ WHO/UNU (2004), and Mifflin-St Jeor equations in 36 young adult women aged 20 to 24 years (BMI 20.7 \pm 1.6 ) and 29 elderly women aged 60 to 76 years (BMI 25.5 \pm 1.6 ). Measures of central tendency, dispersion, and position were calculated for quantitative variables. The Kruskal-Wallis test was used to compare RMR between the different formulae and the Mann-Whitney test was used to compare RMR IC between groups after verifying normality by the Shapiro Wilks test. The statistical significance level was $=0.05$. Results: The Harris-Benedict, FAO/WHO/UNU (2004), FAO/OMS/ UNU (1985), and Mifflin-St Jeor equations showed a statistically significant overestimation ( $p<0.0001)$ of 290.5, 196.8, 200.1, and $188.0 \mathrm{kcal} / \mathrm{d}$ and 220.0, 211.9, 235.8, and $79.4 \mathrm{kcal} / \mathrm{d}$ in young adult and elderly women, respectively. The RMR IC between young adult women $(1050.0 \mathrm{kcal} / \mathrm{d})$ and elderly women $(985,0 \mathrm{kcal} / \mathrm{d})$ exhibited a significant statistical difference ( $p=0.008)$. A higher overestimation was found by the Harris-Benedict test and the FAO/ WHO/UNU (1985) equation in young adult and elderly women, respectively. Conclusions: Predictive equations overestimated RMR in young adult and elderly women. Thus, its routine use could lead to malnutrition due to excess intake.

Key words: Resting metabolic rate, predictive formulae, indirect calorimetry, women, normal nutritional status.
\end{abstract}

\section{INTRODUCCIÓN}

El gasto energético total, considera entre sus principales componentes: la tasa metabólica basal, la termogénesis endógena y la actividad física. La tasa metabólica basal fue definida como la tasa mínima de gasto energético compatible con la vida, y constituye el 60-70\% del gasto energético diario en la mayoría de los adultos sedentarios. Los términos de tasa
Marcela Ruiz De la F. Alejandra Rodríguez F.

Departamento de Nutrición y Salud Pública, Facultad de Ciencias de la Salud y de los Alimentos, Universidad del Bío-Bío, Chillán, Chile.

Dirigir la correspondencia a:

Marcela Ruiz De la Fuente Nutricionista

Magister en Nutrición Clínica Adulto Departamento de Nutrición y Salud Pública Universidad del Bío-Bío, sede Chillán, Chile. Teléfono: (56-42) 2463229

E-mail: marcelaruiz@ubiobio.cl

Este trabajo fue recibido el 15 de Octubre de 2013 y aceptado para ser publicado el 18 de Enero de 2014. metabólica basal (TMB) y tasa metabólica en reposo (TMR) se tienden a utilizar indistintamente, sin embargo, este último aunque si bien igual considera todos los requisitos de la medición de la $T M B$, no requiere ayuno previo; presentando una diferencia entre sí de al menos un 10\% (1).

Entre los principales factores determinantes de la TMR están: el tamaño corporal, la composición corporal, la edad, 
el sexo y la producción de hormonas (2), siendo entre todos ellos la masa magra su principal determinante (3). La TMR puede ser estimada o medido, estimada mediante ecuaciones predictivas y medida mediante: calorimetría indirecta, directa, y agua doblemente marcada. La calorimetría indirecta $(\mathrm{Cl})$ es un método no invasivo y validado para determinar la TMR (4). Se denomina indirecta, por que determina el gasto energético por medio de los equivalentes calóricos del $\mathrm{O}_{2}$ consumido y del dióxido de carbono $\mathrm{CO}_{2}$ producido, cuyas cantidades difieren según el sustrato energético que se esté utilizando. La producción de energía corresponde a la conversión de la energía química contenida en los nutrientes, en energía química almacenada como ATP, y en la energía disipada como calor en los procesos de oxidación. Si se acepta que todo el $\mathrm{O}_{2}$ consumido se utiliza para oxidar los sustratos energéticos (proteínas, carbohidratos y lípidos), y que todo el $\mathrm{CO}_{2}$ producido se elimina por la respiración, es posible calcular la energía total producida por los nutrientes. Este método es un referente como estándar para determinar la TMR en la clínica y en estudios de investigación, sin embargo, no siempre está disponible, debido al elevado costo del equipo y a la necesidad de contar con un personal entrenado. Por consiguiente, las ecuaciones de regresión, usando la calorimetría indirecta como un criterio de medida para desarrollar estos modelos predictivos, son comúnmente usados para estimar la TMR.

Entre las ecuaciones predictivas más utilizadas están: la ecuación de Harris- Benedict, realizada en el laboratorio de Nutrición de Carnegie en Boston y publicada originalmente en el año 1919, basada en mediciones realizadas a 136 hombres de 16 a 63 años y 103 mujeres de 15 a 74 años, con estado nutricional normal; las Ecuaciones de FAO/OMS/UNU año 1985, que utilizaron como base principalmente los datos de los estudios de Shofield realizados en hombres y mujeres de 19 a 82 años, con estado nutricional normal, sobrepeso u obeso. En esta fórmula se ha descrito: limitados datos sobre lactantes, adolescentes y adultos mayores, carencia de datos de personas provenientes de países en desarrollo, poca variabilidad étnica y geográfica, y baja inclusión de individuos de regiones tropicales. Las ecuaciones FAO/OMS/ UNU año 2004, fueron desarrolladas igualmente a partir de la base de datos de Shofield utilizada en la estimación de la TMR (1985) (5). Por último la ecuación de Mifflin St-Jeor, que data del año 1990, se basó en un estudio realizado en 498 sujetos de 19 a 78 años de ambos sexos, que presentaban estado nutricional normal, sobrepeso u obeso, y cuya composición racial no fue especificada. Por su exactitud esta ecuación ha sido recomendada por la Asociación Americana de Dietista (6).

Las ecuaciones predictivas descritas anteriormente, se han asociados a subestimaciones pero principalmente a sobreestimaciones de la TMR (7). La agudeza de las fórmulas predictivas también está determinada por el estado nutricional del individuo, describiéndose en obesos adultos, una disminución en la agudeza, comparado con los sujetos no obesos (6).

Sobrestimar los requerimientos energéticos, contribuye a facilitar un balance energético positivo y por consiguiente la malnutrición por exceso del paciente. La investigación se dividió en dos etapas, en su primera etapa el objetivo fue comparar la TMR medida a través del método de calorimetría indirecta, con algunas ecuaciones predictivas, en mujeres adultas jóvenes y mayores con índice de masa corporal (IMC) normal, y en su segunda etapa, se analizará igualmente su comportamiento en otros estados nutricionales de sobrepeso y obesidad. A continuación se describen los resultados de la primera etapa del estudio.

\section{SUJETOS Y MÉTODO}

El estudio fue analítico, de corte transversal. La muestra estuvo compuesta sólo por participantes del género femenino, 36 mujeres adultas jóvenes de 20-24 años de edad, estudiantes de la Universidad del Bío-Bío sede Chillán, y 29 mujeres adultas mayores de 60-76 años, pertenecientes al Centro de Salud Familiar Violeta Parra de la ciudad de Chillán, Chile, las cuales fueron elegidas aleatoriamente de acuerdo a los criterios de inclusión establecidos: IMC normal es decir de 18.5 a 24.9 en las mujeres jóvenes y de 23.1 a 27.9 en las mujeres adultas mayores, buen estado de salud, ausencia de patología hípermetabólica, peso corporal estable $( \pm 3 \mathrm{Kg})$ en los últimos 6 meses, ausencia de ingesta de medicamentos que modifiquen el gasto energético; y en el caso particular de las mujeres jóvenes, no estar cursando su ciclo menstrual, embarazada o amamantando.

El estudio contó con la aprobación del Comité de Ética de las instituciones respectivas donde fue tomada la muestra, y con el consentimiento informado de las participantes.

Se realizaron las mediciones antropométricas de peso y talla corporal, utilizando los puntos de corte establecidos por la OMS 1998 (8), de todas ellas fueron realizadas por un mismo evaluador de profesión nutricionista. Se determinó el índice de masa corporal o IMC (peso/(talla²), usando los criterios establecidos por la Organización Mundial de la salud (OMS).

La tasa metabólica en reposo (TMR), se midió mediante el método de calorimetría indirecta $(T M R C I)$, utilizando un equipo Datex - Ohweda modelo Deltatrac II, perteneciente a la Unidad de Metabolismo Energético de la Universidad del Bío-Bío de la ciudad de Chillán. A nivel ambiental se controló que la concentración de $\mathrm{CO}_{2}$ fuera menor a $3 \%$ y existiera termo neutralidad ambiental $\left(20-24^{\circ} \mathrm{C}\right)$. El equipo fue auto calibrado antes de cada medición, utilizando una mezcla de gases de $95 \% \mathrm{O}_{2}$ y $5 \% \mathrm{CO}_{2}$.

El día de la medición de la TMR Cl, todas las voluntarias adultas jóvenes fueron instruidas a dirigirse al laboratorio con el mínimo esfuerzo físico posible que pudiese alterar la TMR; particularmente en el caso de las adultas mayores, todas fueron trasladadas desde sus casas en taxi al laboratorio. Previo a la medición de la TMR Cl, se verificó ausencia de antecedentes de enfermedad tiroidea, anemia, y cumplimiento del ayuno previo (10-12 hrs.); también fueron controlados los siguientes signos vitales: temperatura corporal a nivel axilar menor a $37,0{ }^{\circ} \mathrm{C}$, y frecuencia respiratoria entre $12-18$ respiraciones por minuto.

Antes de la medición las mujeres debieron hacer un reposo de 30 minutos, posteriormente, se realizó a cada una la medición de la TMR Cl a las 8:30 hrs. AM y durante un periodo consecutivo de 30 minutos; durante ese periodo la paciente debió estar en posición supina, sin dormir y tranquila. La TMR CI fue calculada por el equipo a partir de la medición del consumo de $\mathrm{O}_{2}$ y eliminación de $\mathrm{CO}_{2}$ y se trabajó con la mediana de los valores arrojados de cada paciente.

La validez del examen se ratificó mediante el valor del cociente respiratorio $(\mathrm{Q} r)$, obtenido de la relación entre el $\mathrm{CO}_{2}$ eliminado y el $\mathrm{O}_{2}$ consumido por el paciente, el que debió mostrar un rango filológico normal entre 0,7-1,0 (9); como también, mediante la verificación de la fluctuación en el intercambio de volumen de $\mathrm{CO}_{2}\left(\mathrm{ml} / \mathrm{min}\right.$.) y de $\mathrm{O}_{2}$, (ml/ 
$\min )(10)$.

Los resultados de la TMR Cl, fueron comparados con la TMR estimada mediante las ecuaciones predictivas de: HarrisBenedict 11, FAO/OMS/UNU año 1985 y FAO/OMS/UNU año 200412 ,13. y de Mifflin St-Jeor. (tabla 1).

Se calculó la diferencia porcentual entre la TMR estimada y la TMR Cl en cada mujer del estudio, mediante la siguiente formula ((TMR -TMR CI)/TMR Cl*100), definiendo la existencia de concordancia entre los valores cuando la diferencia porcentual entre la TMR estimada y la TMR Cl, se encontró dentro de $\pm 10 \%$.

Los datos se analizaron mediante estadística univariada y bivariada. Para la descripción de variables numéricas se calcularon medidas de tendencia central, dispersión y posición, las variables cualitativas se trataron con frecuencia absoluta y porcentajes. Para comparar la TMR entre las diferentes fórmulas, se usó la prueba de Kruskall Wallis, y para comparar la $\mathrm{TMB} \mathrm{Cl}$ entre los dos grupos de mujeres con diferente grupo etarios, la prueba de Mann- Whitney, previa verificación de normalidad de los datos con la prueba de Shapiro Wilks. Se usó un nivel de significancia estadística $\alpha=0,05$.

\section{RESULTADOS}

La población estudiada estuvo constituida por un total de 65 mujeres, 36 adultas jóvenes y 29 adultas mayores. La media del IMC fue $20,7 \pm 1,6$ para el grupo de adultas

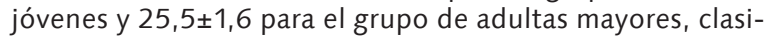
ficando ambos grupos, con un estado nutricional de normal según IMC.

En las adultas jóvenes la TMR estimada mediante $\mathrm{Cl}$ presentó una mediana de 1050,0 Kcal/día, el 25\% $\left(\mathrm{P}_{25}\right)$ de ellas gastó menos de $1007,5 \mathrm{Kcal} /$ día y el $75 \%,\left(\mathrm{P}_{75}\right)$ menos de 1160,0 Kcal/día. Al evaluar la TMR obtenida a través de la fórmula Harris -Benedict, se obtuvo una mediana de 1340,5 $\mathrm{Kcal} /$ día, el $25 \%$ presentó valores menores a 1315,9 kcal/día y el 75\% inferiores a $1385,9 \mathrm{Kcal} /$ día. Con la fórmula FAO/OMS/ UNU año 1985 la mediana fue de 1250,1 Kcal/día, el 25\% de ellas gastó menos de 1205,3 Kcal/día y el 75\% de ellas menos de 1300,0 Kcal/día. Usando la fórmula FAO/OMS/UNU año 2004, los resultados mostraron una mediana de $1246,8 \mathrm{Kcal} /$ día, donde el 25\% de ellas gastó menos de 1201,6 kcal/día y el $75 \%$ menos de $1297,1 \mathrm{Kcal} /$ día. A través de la fórmula de Mifflin St-jeor la mediana de la TMR fue de 1238,0 Kcal/día, el $25 \%$ obtuvo valores menores a $1205,4 \mathrm{Kcal} /$ día y el $75 \%$ inferiores a 1299,2 Kcal/día. La comparación de la TMR fue estadísticamente diferente entre los métodos de estimación usados $(p<0.0001)$. De esta forma, existió para este grupo de mujeres una sobrestimación de la TMR respecto al método de $\mathrm{Cl}(\mathrm{p}<0,0001)$ en las fórmulas de Harris- Benedict, FAO/OMS/ UNU año 2004, FAO/OMS/UNU año 1985 y Mifflin St-Jeor de $290,5,196,8,200,1$ y 188,0 Kcal/días, respectivamente. (figura 1).

La medición de la TMR en mujeres adultas mayores, mediante $\mathrm{Cl}$ fue estadísticamente diferente de la estimación mediante los demás métodos $(\mathrm{p}<0,0001)$. De esta manera, con $\mathrm{Cl}$ se obtuvo una mediana de $985,0 \mathrm{Kcal} / \mathrm{día}$, el $25 \%$ de la muestra gastó bajo las 940,0 Kcal/día y el $75 \%$ menos de $1075,0 \mathrm{Kcal} /$ día. Mediante la fórmula Harris - Benedict, la mitad de las mujeres se clasificaron con 1205,0 Kcal/día, el $25 \%$ gastó bajo las $1153,9 \mathrm{Kcal} /$ día y el $75 \%$ menos de 1238,3 $\mathrm{Kcal} /$ día. Con la fórmula de la FAO/OMS/UNU año 1985 la mediana fue de 1220,8 kcal/día, el 25\% de la muestra gastó bajo $1205,0 \mathrm{kcal} /$ día y el $75 \%$ bajo de $1238,3 \mathrm{Kcal} /$ día. El $50 \%$ de las mujeres mediante la fórmula FAO/OMS/UNU año 2004 clasificó con 1198,9 Kcal/día, el 25\% gastó bajo 1185,3 $\mathrm{Kcal} /$ día y el $75 \%$ bajo $1233,4 \mathrm{Kcal} /$ día. Por último a través de la fórmula de Mifflin St-Jeor la mitad de la muestra obtuvo $1064,4 \mathrm{Kcal} /$ día, el 25\% gastó menos de 1004,8 kcal/día y el $75 \%$ bajo $1118,7 \mathrm{kcal} /$ día. Al igual que el grupo de adultas jóvenes, el de adultas mayores también evidenció una sobreestimación estadísticamente significativa de la TMR estimada mediante ecuaciones predictivas respecto a la medida por $\mathrm{Cl}(\mathrm{p}<0.0001)$. Las fórmulas de Harris-Benedict, FAO/OMS/ UNU año 2004, FAO/OMS/UNU año 1985 y fórmula Mifflin St-Jeor, sobreestimaron en 220,0, 211,9, 235,8 y 79,4 Kcal/ días, respectivamente. (figura 2). Por lo tanto, en las adultas jóvenes la fórmula que más sobrestimó la TMR $\mathrm{Cl}$, fue la de Harris - Benedict, y en el caso de las adultas mayores, la de FAO/OMS/UNU año 1985.

Al comparar la mediana de la TMR CI de 1050,0 Kcal/día y de 985,0 Kcal/día entre las mujeres jóvenes y adultas mayores, respectivamente, se observó una diferencia estadísticamente significativa $(p=0,008)$.

El análisis de concordancia mostró en las adultas jóvenes un mayor grado de precisión con las fórmulas de la FAO/ OMS/UNU año 1985 y año 2004, donde en ambas el 30,5\% de las estimaciones oscilaron en $\pm 10 \%$ de la TMR Cl; en el caso de las adultas mayores, la ecuación de Mifflin St- Jeor

TABLA 1

Ecuaciones predictivas para estimar gasto energético en reposo

\begin{tabular}{|c|c|c|c|c|c|}
\hline Ecuación & Sujetos & Estado nutricional & Edad años & \multicolumn{2}{|c|}{ Fórmula para sexo femenino } \\
\hline $\begin{array}{l}\text { Harris-Benedict } \\
\text { (1919) }\end{array}$ & 103 & $\mathrm{~N}$ & $15-74$ & \multicolumn{2}{|c|}{$9,563 \times P)+(1,84 \times T)-(4,676 \times E)+655,09$} \\
\hline $\begin{array}{c}\text { Mifflin-ST Jeor } \\
\text { (1990) }\end{array}$ & 498 & $\mathrm{~N}, \mathrm{Sp}, \mathrm{O}$ & $19-78$ & \multicolumn{2}{|c|}{$(9,99 \times P)+(6,25 \times T)-(4,92 \times E)-161$} \\
\hline $\begin{array}{c}\text { FAO/OMS/UNU } \\
\text { (1985) }\end{array}$ & 247 & $\mathrm{~N}, \mathrm{Sp}, \mathrm{O}$ & $19-82$ & $\begin{array}{l}14,7 \times P+496 \\
10,5 \times P+596\end{array}$ & $\begin{array}{c}\text { (18- 30años) } \\
\text { (> } 60 \text { años) }\end{array}$ \\
\hline $\begin{array}{l}\mathrm{FAO} / \mathrm{OMS} / \mathrm{UNU} \\
\text { (2004) }\end{array}$ & 247 & $\mathrm{~N}, \mathrm{Sp}, \mathrm{O}$ & $19-82$ & $\begin{array}{l}14,818 \times P+886,6 \\
9,082 \times P+658,5\end{array}$ & $\begin{array}{l}\text { (18-30 años) } \\
\text { (> } 60 \text { años) }\end{array}$ \\
\hline
\end{tabular}

P: peso corporal (Kg); T: talla (cm), E: edad (años); N: normal; SP: sobrepeso; O: obesidad. 


\section{FIGURA 1}

Comparación de la tasa metabólica en reposo según calorimetría indirecta versus ecuaciones predictivas, en mujeres jóvenes de peso corporal normal.

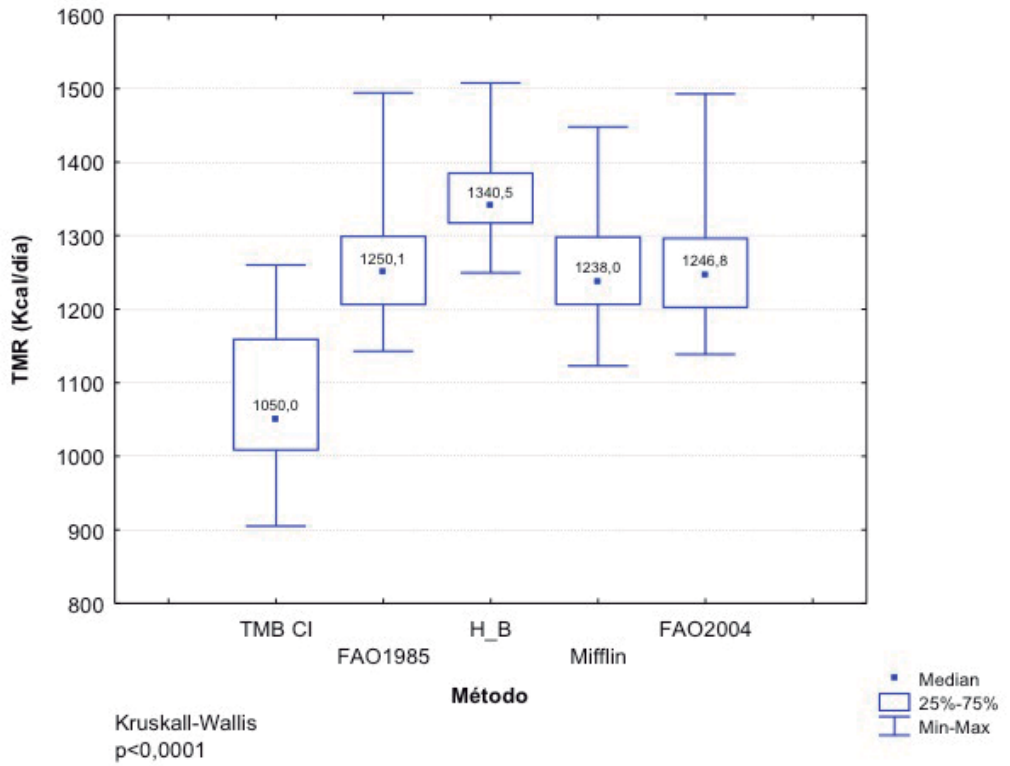

FIGURA 2

Comparación de la tasa metabólica en reposo según calorimetría indirecta versus ecuaciones predictivas, en adultas mayores de peso corporal normal.

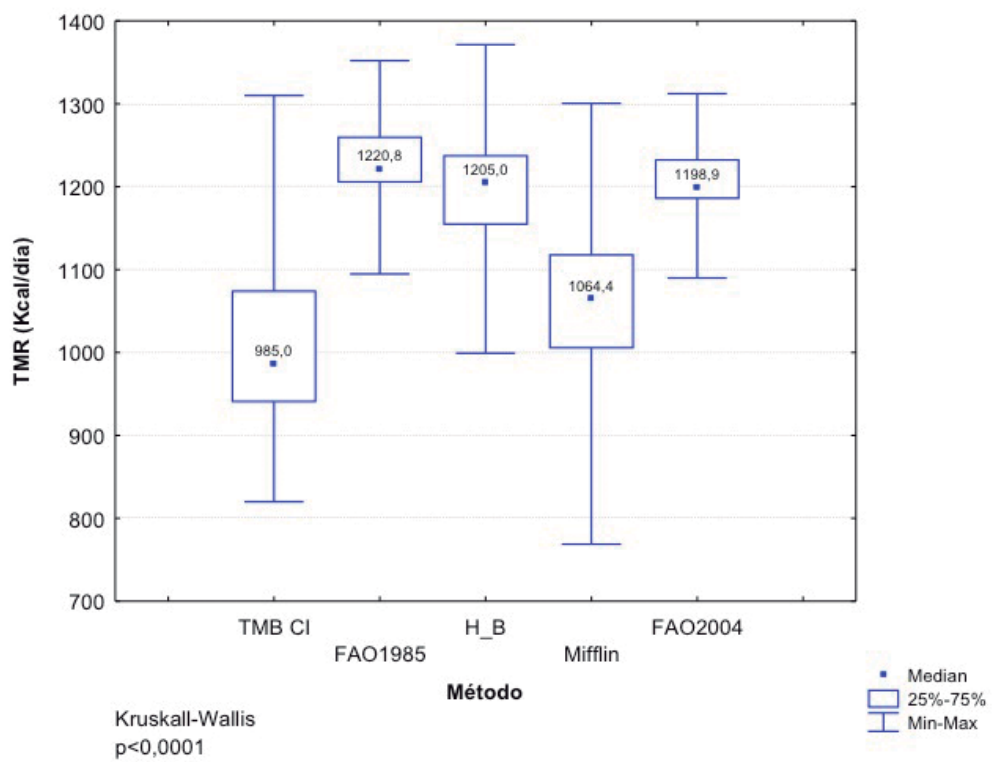


fue la que presentó una mayor precisión en un 56,6\% de las estimaciones. (tabla 2).

\section{DISCUSIÓN}

La agudeza en la estimación de la TMR mediante el uso de las ecuaciones predictivas, varía dependiendo de algunas variables tales como: género, IMC, edad, raza o etnicidad (14). Se ha descrito en ellas errores de subestimación como sobrestimación de la TMR estimada, con una mayor agudeza en la estimación en adultos no obesos en relación a obesos (6).

La TMR calculada mediante las ecuaciones predictivas respecto a la TMR medida por calorimetría indirecta, mostró una sobreestimación estadísticamente significativas, tanto en adultas jóvenes como en adultas mayores ( $P>0,0001)$. Situación también reportada en mujeres adultas Vietnamitas con IMC normal, donde la fórmula FAO/OMS/UNU año 1985 (15) sobreestimó en un 9\%, y en mujeres adultas mayores, donde la fórmula Harris-Benedict como de la FAO/OMS/UNU año 1985 , sobreestimaron en un $12 \%$ (16). A nivel nacional las ecuaciones predictivas de Harris- Benedict y de la FAO/OMS/ UNU año 1985, también han mostrado sobreestimación de la TMR $(17,18)$.

La fórmula Harris - Benedict es una de las ecuaciones más ampliamente usadas por clínicos en Canadá, Estados Unidos (19), y también en nuestro país. Fue originalmente validada en 239 sujetos blancos de peso corporal normal, de los cuales 103 fueron mujeres (11). En las adultas jóvenes esta ecuación presentó la mayor sobreestimación en más de 290,5 $\mathrm{Kcal} /$ día en relación a la TMR Cl, situación también reportada en otros estudios internacionales, uno realizado en mujeres canadienses jóvenes (20), y otro realizado en mujeres chinas jóvenes y saludables (21). Por el contrario, la ecuación de Mifflin St-Jeor recomendada por la asociación Americanas de Dietistas, por su mejor exactitud y menor magnitud de error, fue la que presentó la menor sobreestimación además de una buena concordancia, surgiendo como una alternativa de ecuación predictiva a utilizar, previa validación en un mayor número de individuos.

La fórmula FAO/OMS/UNU año 1985 en mujeres adultas mayores, fue la que mostró la mayor sobreestimación de la TMR Cl, en 235,8 Kcal/día, situación también descrita en otra investigación (14)

Esta ecuación, se basó en los datos de Scholfield que comprendió 114 estudios publicados y 7173 mediciones de la $T M R$, realizadas en sujetos norteamericanos y europeos. El $47 \%$ de la base de datos estuvo conformada por policías Italianos, donde se ha descrito una mayor TMR por kilogramo de peso corporal, en comparación a los otros individuos participantes (22). Por lo tanto, los resultados encontrados en la investigación podrían explicarse por la diferencia étnica entre la población estudiada y la población que derivó la ecuación. La ecuación de Mifflin St-Jeor igualmente en este grupo mostró la menor sobreestimación de la TMR Cl y mejor concordancia, sin embargo, es importante señalar que en algunos casos también mostró subestimación, situación descrita en otras investigaciones (6).

La edad se asocia con una progresiva declinación de la TMR de 1-2\% por década después de los 20 años de edad (23), relacionada a la disminución de la masa magra y a cambios en la distribución de la grasa corporal, observada con el paso de los años (24), el cual es coherente con las diferencias encontradas entre los grupos analizados, donde las adultas mayores presentaron menores valores de la $\mathrm{TMR} \mathrm{Cl}$, comparadas con las adultas jóvenes $(p=0,008)$.

Cuando la ingesta energética supera el requerimiento, el exceso de energía ingerido es depositado en el cuerpo. De acuerdo a lo anterior se ha descrito que un balance energético positivo de $6600-8000 \mathrm{kcal}$, facilita el incremento de 1 kilogramo de peso corporal (25). De este modo, la sobreestimación de los requerimientos energéticos obtenida mediante el cálculo de las ecuaciones predictivas, puede contribuir en el mediano a largo plazo, a un balance energético positivo y por ende a una malnutrición por exceso en poblaciones en riesgo. Por consiguiente, se hace necesario la realización de más investigaciones a nivel nacional donde se determine los requerimientos energéticos de nuestra población, en los diferentes grupos etarios y estados nutricionales, que permitan finalmente la formulación de ecuaciones predictivas de mayor exactitud para nuestra gente.

\section{RESUMEN}

Introducción: La tasa metabólica en reposo (TMR) puede ser medida por calorimetría indirecta (TMR CI) o estimada mediante ecuaciones predictivas, que pueden subestimar o sobreestimar los requerimientos energéticos. Objetivo: Comparar la TMR medida por calorimetría indirecta, con la estimadas

TABLA 2

Concordancia en rango de $\pm 10 \%$ entre TMR medido y la TMR estimado por fórmulas.

\begin{tabular}{|c|c|c|c|c|}
\hline Ecuación & Grupo etario & $\begin{array}{l}\% \text { sujetos dentro } \\
\text { rango concordancia }\end{array}$ & $\begin{array}{l}\% \text { sujetos sobre } \\
\text { rango concordancia }\end{array}$ & $\begin{array}{l}\% \text { sujetos bajo } \\
\text { rango concordancia }\end{array}$ \\
\hline \multirow[t]{2}{*}{ Harris - Benedict } & Mujeres jóvenes & 11,1 & 88,8 & 0 \\
\hline & Mujeres adultas mayores & 26,6 & 73,3 & 0 \\
\hline FAO/OMS/UNU & Mujeres jóvenes & 30,5 & 69,4 & 0 \\
\hline 1985 & Mujeres adultas mayores & 26,6 & 73,3 & 0 \\
\hline FAO/OMS/UNU & Mujeres jóvenes & 30,5 & 69,4 & 0 \\
\hline 2001 & Mujeres adultas mayores & 26,6 & 73,3 & 0 \\
\hline \multirow[t]{2}{*}{ Mifflin St- Jeor } & Mujeres Jóvenes & 27,7 & 72,2 & 0 \\
\hline & Mujeres adulta mayores & 56,6 & 43,3 & 0 \\
\hline
\end{tabular}


por ecuaciones predictivas, en adultas jóvenes y mayores con índice de masa corporal (IMC) normal. Sujetos y métodos: Estudio analítico, transversal. Se midió la TMR CI y se estimó a través de las ecuaciones Harris-Benedict 1919; FAO/OMS/ UNU 1985; FAO/OMS/UNU 2004, y Mifflin St -Jeor, en 36

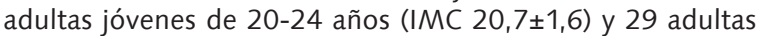
mayores de 60-76 años (IMC 25,5 $\pm 1,6$ ). Para variables numéricas se calcularon medidas de tendencia central, dispersión y posición. Para comparar la TMR entre las diferentes fórmulas, se usó la prueba de Kruskall Wallis, y para comparar la $\mathrm{TMB} \mathrm{Cl}$ entre grupos, la prueba de Mann- Whitney, previa verificación de normalidad con prueba Shapiro Wilks. Se usó nivel de significancia estadística $\alpha=0,05$. Resultados: Las ecuaciones Harris-Benedict, FAO/OMS/UNU 2004, FAO/OMS/UNU 1985 y Mifflin St-Jeor, mostraron sobrestimación estadísticamente significativa ( $p<0.0001$ ) de 290,5, 196,8, 200,1 y 188,0 kilocalorías/día en adultas jóvenes, y de 220,0, 211,9, 235,8 y 79,4 kilocalorías/días, en adultas mayores, respectivamente. La TMR Cl entre adultas jóvenes (1050,0 kcal/día) y mayores (985,0 kcal/día), presentó una diferencia estadísticamente significativa $(p=0,008)$. En adultas jóvenes Harris - Benedict mostró mayor sobrestimación y en adultas mayores la FAO/ OMS/UNU 1985. Conclusiones: Las ecuaciones predictivas, sobrestimaron la TMR en adultas jóvenes y mayores, por lo cual su uso rutinario, podría facilitar la malnutrición por exceso.

Palabras clave: Tasa metabólica reposo, fórmulas predictivas, calorimetría indirecta, mujeres, estado nutricional normal.

Agradecimientos: Se agradece al Centro de Salud Familiar Violeta Parra de la Ciudad de Chillán y a los alumnos de la Carrera de Nutrición y Dietética de la Universidad del Bío-Bío, quienes hicieron posible el desarrollo de esta investigación. También al proyecto código 102520 2/R de la Universidad del Bío-Bío.

\section{BIBLIOGRAFÍA}

1. Mataix J, Martinez JA. Balance de energía corporal. En : Nutrición y alimentación humana. Mataix J Ed. Oceano/ Ergon. Barcelona. 2006:703-22.

2. Maha $n$ LK, Escott-Stump S. Dietoterapia de Krause. Edit. Elsevier Masson. 12 a ed. Barcelona 2009:22-37.

3. Esteves de Oliveira FC, de Mello Cruz AC, Goncalves Oliveira C, Rodriguez Ferreira Cruz AC, Mayumi Nakajima $V$, Bressan J. Gasto energético de adultos brasileños saludables: una comparación de métodos. Nutr Hosp. 2008; $23: 554-61$.

4. Coper JA, Watrs AC, O' Briend MJ, et al. Assesing validity and reliability of resting metabolic rate in six gas analysis systems. J Am Diet Assoc 2009; 109(1):128-32.

5. Vargas $M$, Lancheros $L$, Barrera MP. Gasto energético en reposo y composición corporal en adultos. Rev Fac Med. 2011; 59 (Supl 1):S43-58.

6. Frankenfield D, Roth-Yousey L, Compher C.Comparison of predictive equations for resting metabolic rate in healthy nonobese and obese adults: a systematic review. J. Am Diet Assoc. 2005;105(5):775-89.

7. Muller MJ, Bosy-Westpha AL, Klau S, Kreymann G, Luhrmann PM, Neuhauser-Berthold M, et al. World Health Organization equations have shortcomings for predicting resting energy expenditure in person from a moderm, affluent population: generation of a new reference standard from a retrospective analysis of a German database of resting energy expenditure. Am J Clin Nutr.
2004; 80: 1379-90.

8. World Healdth Organization (WHO). Obesity: preventing and managing the global epidemic. Geneva: World Health Organization; 1998.

9. McClave SA, Lowen CC, Kleber MJ, McConnell JW, Jung $L Y$, Goldsmith LJ. Clinical use of the respiratory quotient obtained from indirect calorimetry. JPEN. 2003; 27: 21-6.

10. Boullata J,Williamns J, Cottrell F at el. Accurate Determination of energy need in hospitalizad patient. J Am Diet Assoc. 2007; 107:393-401.

11. Harris JA, Benedict FG:" A Biometric study of basal metabolism in man". Washington. DC: Carnegie Institution of Washington, 1919.

12. FAO/OMS/UNU. Neccesidades de energía y proteínas. Informe de una Reunión Consultiva Conjunta FAO/OMS/ UNU de Expertos. OMS, genebra, 1985.

13. FAO/WHO/UNU. Expert Comsultation. Interim ReportReport on Humman Energy Requeriments, 2001.

14. Hasson $R$, Howe $C H$, Jones $B$, Freedson P. Accuracy of four resting metabolic rate prediction equations: Effects of sex, body mass index, age, and race/ethnicity. I Sc Med Sport. 2011; 14; 344-51.

15. B T Nhung, N C Khan, LT Hop, D T K Lien, D S N T Le, V TT Hien, D Kunii, T Sakai, M Nakamori and S Yamamoto. FAO/WHO/UNU equations overestimate resting metabolic rate in Vietnamese adults. European J Clin Nutr. 2005; 59, 1099-104.

16. Arciero PJ, Goran MI, Gardner AM, Ades PA, Tyzbir RS, Poehlman ET. A practical equation to predict resting metabolic rate in older females. J Am Geriatr Soc. 1993; 41:389-95.

17. Carrasco F, Reyes Eliana, Núñez CH, Reidemann S, Rimler $O$, Sánchez G, Sarrat G. Gasto energético de reposo medido en obesos y no obesos: comparación con la estimación por fórmulas y ecuaciones propuestas para la población chilena. Rev. Méd. Chile. 2002, 130 (1):51-60.

18. López $M$, Valenzuela $E$. Nuevas fórmulas para mejorar la estimación del gasto energético basal en adultos de Chillán. Rev Chil Nutr. 2007; 34(4), 338-45.

19. Reeves MM. Capra S: Predicting energy requeriments in the clinical setting: are current methods evidence based? Nutr Rev 2003,61:143-151.

20. Basc A, Tereszkowski C, Eddwards M et al. Published predictive equations overestimate measured resting metabolic rate in young, healthy females. J Am Coll Nutr. 2010;29(3): 222-7

21. Rao ZY, Wu XT, Lian BM, Wang MY, Hu W. Comparison of five equations for estimating resting energy expenditure in Chinese young, normal weight healthy adults. Eur J Med Res. 2012; 1:17:26

22. Henry CJK. Basal metabolic rate studie in humans: measurement and development of new equations. Public Health Nutrition. 2005, 8 (7A):1133-1152.

23. Elia, M., Ritz, P., et al. Total energy expenditure in the elderly. Eur J Clin Nutr. 2000;54 (Suppl. 3), S92-103.

24. Luhrmann PM, Edelmann-Schafer B, Neuhauser-Berthold $M$. Changes in resting metabolic rate in an elderly German population: cross-sectional and longitudinal data. J Nutr Health Aging. 2010; 14(3): 232-6.

25. Diaz E, Pretince A M, Goldberg CR, Murgatryd PR, Coward Wa. Metabolic response to experimental overfeeding in lean and overweight healthy volunteers. Am J Clin Nutr. 1992; 56:641-55. 